\title{
MicroRNA-26b-5p regulates cell proliferation, invasion and metastasis in human intrahepatic cholangiocarcinoma by targeting $\mathrm{S100A7}$
}

\author{
FEI FAN ${ }^{1 *}$, JIONGJIONG LU ${ }^{1 *}$, WENLONG YU ${ }^{2}$, YONGJIE ZHANG ${ }^{2}$, SUQIAN XU ${ }^{1}$, \\ LEILEI PANG ${ }^{3}$ and BIN ZHU ${ }^{1}$ \\ Departments of ${ }^{1}$ Special Treatment and ${ }^{2}$ Biliary Surgery II, Eastern Hepatobiliary Surgical \\ Hospital, Second Military Medical University, Shanghai 200438; ${ }^{3}$ Department of Pathology, \\ Maternal and Child Health Hospital of Weihai, Weihai, Shandong 264200, P.R. China
}

Received February 26, 2016; Accepted July 17, 2017

DOI: $10.3892 / \mathrm{ol} .2017 .7331$

\begin{abstract}
The present study aimed to investigate the effects of microRNA expression in intrahepatic cholangiocarcinoma (ICC). It was identified that the expression of microRNA-26b-5p (miR-26b-5p) was downregulated in ICC tissues compared with matched adjacent non-tumor tissues. Furthermore, miR-26b-5p expression was downregulated in metastatic ICC tumor tissues and invasive ICC cell line subpopulations compared with non-metastatic tumor tissue and the parental ICC cells. In vitro studies demonstrated that transfection with an miR-26b-5p mimic inhibited the proliferation, migration and invasion of RBE and HCCC-9810 cells, whereas an miR-26b-5p inhibitor promoted these abilities. S100 calcium-binding protein A7 (S100A7) was predicted as a direct target of miR-26b-5p. Transfection with an miR-26b-5p mimic decreased S100A7 expression, whereas an miR-26b-5p inhibitor increased S100A7 expression. The result of a dual luciferase reporter assay also indicated this interaction. S100A7 was therefore confirmed as a direct target of miR-26b-5p in ICC. The knockdown of S100A7 abrogated the effect of miR-26b-5p on cell migration in RBE and HCCC-9810 cells. In conclusion, the present study demonstrated that miR-26b-5p suppresses the proliferation, migration and invasion of ICC cells by suppressing S100A7.
\end{abstract}

Correspondence to: Professor Bin Zhu, Department of Special Treatment, Eastern Hepatobiliary Surgical Hospital, Second Military Medical University, 225 Changhai Road, Shanghai 200438, P.R. China E-mail: bbinzhu@sina.com

*Contributed equally

Key words: intrahepatic cholangiocarcinoma, microRNA-26b-5p, proliferation, migration, invasion, $\mathrm{S} 100$ calcium-binding protein A7

\section{Introduction}

Intrahepatic cholangiocarcinoma (ICC), the second most common intrahepatic primary tumor after hepatocellular carcinoma (HCC), exhibits a highly invasive nature and frequently metastasizes to the lymph nodes (1). Although progress has been made in the diagnosis and treatment of ICC, the long-term prognosis of patients with ICC remains unsatisfactory (2-4). At present, the treatment for this disease is curative resection, which is associated with a low 5-year survival rate (between 25 and $35 \%$ ) and a high recurrence rate $(67.9 \%)(5,6)$. Furthermore, there have been few effective diagnostic and prognostic molecular markers reported for ICC. A comprehensive understanding of the mechanisms of ICC metastasis will contribute to the identification of novel diagnosis and therapeutic targets.

MicroRNAs (miRNAs/miRs) are 18-25 bp, non-coding, single-strand RNAs that regulate gene expression at the post-transcriptional level by binding to the 3 ' untranslated region (3'UTR) of target mRNAs $(7,8)$. miRNAs are involved in a variety of basic physiological and pathological processes, including cell proliferation, differentiation, metabolism and apoptosis $(9,10)$. Previous studies have demonstrated that miRNAs are frequently downregulated or dysregulated in a range of types of malignancy, including ICC, breast cancer and colorectal cancer, potentially acting as oncogenes or tumor suppressor genes (9-11). Emerging evidence has demonstrated that the aberrant expression of miRNA may serve key roles in the initiation and progression of ICC and HCC (11-13). However, the exact mechanisms by which miRNAs regulate the metastasis of ICC are poorly characterized.

The present study aimed to investigate the effects of miR-26b-5p expression in ICC. miR-26b-5p was identified as downregulated in ICC, particularly in ICC with lymphatic metastasis (mICC) and in invasive subpopulations of the RBE and HCCC-9810 cell lines (iRBE and iHCCC-9810). It was identified that miR-26b-5p mimics inhibited the proliferation, migration and invasion of RBE and HCCC-9810 cells in vitro. Furthermore, S100 calcium-binding protein A7 (S100A7), which is implicated in tumorigenesis, was demonstrated to 
be a direct target of miR-26b-5p. Results of the present study revealed that miR-26b-5p hindered the proliferation, migration and invasive abilities of ICC cells by inhibiting S100A7 expression.

\section{Materials and methods}

Clinical samples. A total of 20 ICC histopathologically verified tumor samples, together with their matched adjacent non-tumor tissues and an additional 40 tumor samples, including $20 \mathrm{mICC}$ samples and 20 samples without detected metastasis, were obtained from patients that underwent resective surgery for ICC or mICC (age range, 35-85 years, mean \pm standard deviation (SD), 67 $\pm 11 ; 25$ female and 35 male samples; 31 cases of stage I-II, 29 cases of stage III according to the grading standards from Chinese Society of Liver Cancer (14) at the Eastern Hepatobiliary Surgical Hospital between September 2012 and May 2014. The tissues were snap frozen in liquid nitrogen immediately subsequent to surgical removal and stored at $-70^{\circ} \mathrm{C}$ until required. The use of all specimens in the present study was approved by the Institutional Ethics Committee of the Second Military Medical University (Shanghai, China). All patients signed informed consent forms for the use of their data and surgical specimens in the present study.

Cell lines and growth conditions. Human ICC RBE and HCCC-9810 cell lines were purchased from Thermo Fisher Scientific, Inc. (Waltham, MA, USA). All cells were cultured in Dulbecco's modified Eagle's medium (DMEM; Invitrogen; Thermo Fisher Scientific, Inc.) supplemented with $10 \%$ fetal bovine serum (FBS; Gibco; Thermo Fisher Scientific, Inc.), $100 \mathrm{IU} / \mathrm{ml}$ penicillin (Gibco; Thermo; Fisher Scientific, Inc.) and $100 \mu \mathrm{g} / \mathrm{ml}$ streptomycin (Gibco; Thermo Fisher Scientific, Inc.) in a humidified $5 \% \mathrm{CO}_{2}$ cell incubator at $37^{\circ} \mathrm{C}$.

Establishment of invasive cell lines. Highly invasive subpopulations from the RBE and HCCC-9810 cell lines were established with 10 rounds of selection from a Matrigel-coated Transwell assay. All Matrigel and supplements were purchased from BD Biosciences (Franklin Lakes, NJ, USA). In brief, the inserts were coated with $50 \mathrm{ml}$ of $1 \mathrm{mg} / \mathrm{ml}$ Matrigel matrix and $1 \times 10^{5}$ cells in $200 \mathrm{ml}$ serum-free DMEM were seeded in the Transwell upper chambers. A total of $600 \mathrm{ml}$ DMEM with $10 \%$ fetal bovine serum was added to the lower chamber. Following incubation for $12-72 \mathrm{~h}$ at $37^{\circ} \mathrm{C}$, the cells that migrated through the Matrigel membranes and attached to the lower surface were further passaged in subsequent sub-clone cultures. The selection of ICC cells was repeated 10 times to establish highly invasive sub-lines. These were designated as iRBE and iHCCC-9810 cells.

RNA isolation and reverse transcription-quantitative polymerase chain reaction ( $R T-q P C R)$. Total RNA was extracted from cultured cells and tissues using TRIzol RNA isolation reagent (Invitrogen; Thermo Fisher Scientific, Inc.). For the reverse transcription, all reagents were purchased from Applied Biosystems (Thermo Fisher Scientific, Inc.). Mature miRNAs were reverse-transcribed from total RNA using specific miRNA RT-primers from the TaqMan Reverse MicroRNA Transcription kit (Applied Biosystems; Thermo Fisher Scientific, Inc.). The temperature protocol for RT was as follows: $16^{\circ} \mathrm{C}$ for $30 \mathrm{~min}, 42^{\circ} \mathrm{C}$ for $30 \mathrm{~min}$ and $85^{\circ} \mathrm{C}$ for $2 \mathrm{~min}$, and finally $4^{\circ} \mathrm{C}$ hold. Following this, pre-amplification step was conducted according to the protocol of TaqMan Reverse MicroRNA Transcription kit (Applied Biosystems; Thermo Fisher Scientific, Inc.). Following the RT process, the pre-amplification process was performed. The temperature cycles were as follows: $95^{\circ} \mathrm{C}$ for $10 \mathrm{~min}, 55^{\circ} \mathrm{C}$ for $2 \mathrm{~min}$ and $72^{\circ} \mathrm{C}$ for $2 \mathrm{~min}$, then 12 cycles of $95^{\circ} \mathrm{C}$ for $15 \mathrm{sec}, 60^{\circ} \mathrm{C}$ for $4 \mathrm{~min}$ and $99.9^{\circ} \mathrm{C}$ for $10 \mathrm{~min}$, and then $4^{\circ} \mathrm{C}$ hold.

Following the RT and pre-amplification process, qPCR was performed using the TaqMan miRNA assay kit primers (Thermo Fisher Scientific, Inc.) with the TaqMan Universal PCR Master mix (Thermo Fisher Scientific, Inc.) and analyzed using an ABI 7500 system (Applied Biosystems; Thermo Fisher Scientific, Inc.). SYBR Premix Ex Taq (Perfect Real Time; Takara Biotechnology Co., Ltd.) was used for qPCR. The temperature protocol for qPCR was as follows: $95^{\circ} \mathrm{C}$ for $10 \mathrm{~min}$, followed by 40 cycles of $95^{\circ} \mathrm{C}$ for $15 \mathrm{sec}$ and $60^{\circ} \mathrm{C}$ for $1 \mathrm{~min}$. To normalize mRNA expression, $\beta$-actin was used as the endogenous internal control with the $2^{-\Delta \Delta \mathrm{Cq}}$ method. The primers for S100A7 were as follows: Forwards, 5'-AACTTC CTTAGTGCCTGTG-3'; reverse, 5'-TGGTAGTCTGTGGCT ATGTC-3'. The primers for $\beta$-actin were: Forward, 5'-AGA GCTACGAGCTGCCTGAC-3'; reverse, 5'-AGCACTGTG TTGGCGTACAG-3'. For all figures of RT-qPCR data, values on the $y$-axis are equal to $2^{\Delta \Delta \mathrm{Cq}}(15)$. Gene expression data were obtained in triplicate in three independent experiments.

Cell transfection. miRNA vectors were purchased from Shanghai GenePharma Co., Ltd. (Shanghai, China), including an miR-26b-5p expression vector (catalog no. mh40312; Applied Biological Materials Inc., Richmond, BC, Canada), a miR-26b-5p control vector (cat. no. mm30393; Applied Biological Materials Inc., Richmond, BC, Canada), miR-26b-5p inhibitor (catalog no. MIN0000083; Qiagen China Co., Ltd., Shanghai, China) and miR-26b-5p mimic (catalog no. MSY0000083; Qiagen China Co., Ltd.). cDNA for the miR-26b-5p precursor (pre-miR-26b-5p) was synthesized by Shanghai GenePharma Co., Ltd. based the sequence information for harmiR-26b-5p (MI0000083) from the miRBase database (www.mirbase.org/cgi-bin/mature.pl?mature acc=MIMAT0000083), which was sub-cloned into a lentiviral expression vector by Shanghai Sunbio Biotechnology Co., Ltd. (Shanghai, China).

The S100A7 cDNA was purchased from Sino Biological, Inc. (Beijing, China) and packaged into a lentiviral expression vector by Shanghai Sunbio Biotechnology Co., Ltd. S100A7 Pre-design Chimera RNAi was purchased from Abnova Co. Ltd. (Taoyuan, Taiwan). The wild-type 3'UTR for human S100A7 was sub-cloned into a firefly/Renilla Dual-Luciferase reporter vector by GeneCopoeia, Inc. (Rockville, MD, USA). A point mutation was produced within the predicted miR-26b-5p interaction region of the S100A7 3'UTR fragments using a Fusion Site Directed Mutagenesis kit from Thermo Fisher Scientific, Inc. The mutated and wild-type 3'UTR region of human S100A7 were each cloned into the 3'UTR position of the previously constructed S100A7 
expression vector. The aforementioned vectors $(1 \mu \mathrm{g} / \mathrm{ml})$ were transfected into ICC cells using Lipofectamine 2000 (Invitrogen; Thermo Fisher Scientific, Inc.) according to the manufacturer's protocol.

Determination of cell proliferation. The transfected cells were seeded on 96-well plates at a density of $1 \times 10^{4}$ cells/well. The plates were incubated at $37^{\circ} \mathrm{C}$ for $48 \mathrm{~h}$, and MTT solution ( $20 \mu \mathrm{l}, 5 \mathrm{mg} / \mathrm{ml}$ ) was added to each well (to a total volume of $250 \mu \mathrm{l})$. Following incubation for $3 \mathrm{~h}$ at $37^{\circ} \mathrm{C}$, the MTT solution was removed and the formazan precipitates were dissolved in $200 \mu \mathrm{l}$ dimethyl sulfoxide and incubated for $30 \mathrm{~min}$ at $37^{\circ} \mathrm{C}$. The absorbance was measured at $540 \mathrm{~nm}$. The experiment was performed in triplicate.

Migration and invasion assays. For the Transwell migration assays, $1 \times 10^{4}$ cells were plated in the top chamber containing a non-coated membrane (24-well insert; $8 \mathrm{~mm}$ pore size; BD Biosciences). For the invasion assays, $2 \times 10^{5}$ cells were plated in the top chamber containing a Matrigel-coated membrane (24-well insert; $8 \mathrm{~mm}$ pore size; BD Biosciences) with serum free DMEM. DMEM supplemented with 10\% (v/v) FBS was used as a chemoattractant in the lower chamber. At $16 \mathrm{~h}$, the non-migrated/non-invading cells were removed from the upper sides of the Transwell membrane filter inserts using cotton-tipped swabs. The migrated/invaded cells on the lower sides of the inserts were stained with Giemsa (3\% working solution) for $30 \mathrm{~min}$ at $37^{\circ} \mathrm{C}$ and the cells were counted using a light microscope (CKX41; Olympus Corporation, Tokyo, Japan). And the experiment was repeated for 3 times.

Luciferase reporter assay. For the prediction of miRNA targets, TargetScan (heep://www.targetscan.org) and miRanda (http://www.microrna.org) were used. For the luciferase reporter assay, RBE cells were respectively transfected with wild-type and mutant firefly/Renilla Dual-Luciferase reporter vectors, as previously described, and subsequently transduced with the miR-26b-5p mimic or inhibitor. The cells were collected following incubation for $48 \mathrm{~h}$. A dual-luciferase reporter assay system (Promega Corporation, Madison, WI, USA) was used to measure Renilla luciferase activity, according to the manufacturer's protocol. The luciferase reporter assay was repeated three times.

Statistical analysis. The quantitative data are expressed as the mean \pm SD. GraphPad Prism 5 software (GraphPad Software, Inc., La Jolla, CA, USA) was used for statistical analysis. Comparisons between two or more groups were subjected to a two-tailed Student's t-test or one-way analysis of variance followed by least significant difference test. $\mathrm{P}<0.05$ was considered to indicate a statistically significant difference.

\section{Results}

Candidate miRNA selection. In order to identify novel biomarkers for ICC, 5 candidate miRNAs (miR-101-3p, miR-147a, miR-196-5p, miR-93-3p and miR-26b-5p), which have been associated with other types of cancer and diseases (16-20), were quantified in RBE, iRBE, HCCC-9810

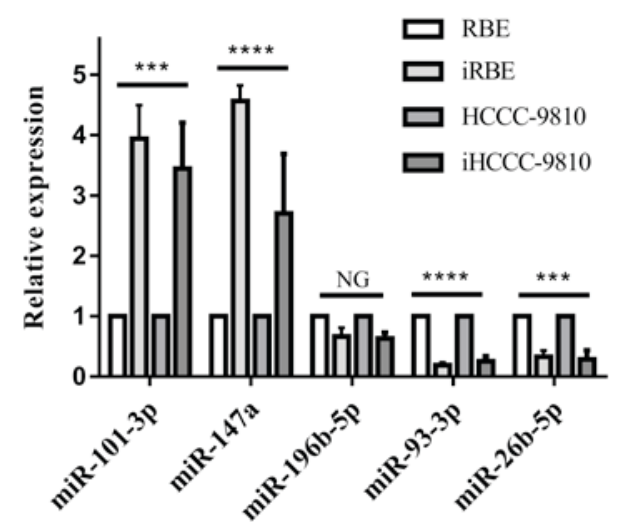

Figure 1. Expression of five candidate miRNAs in intrahepatic cholangiocarcinoma cell lines. miR/miRNA, microRNA; iRBE, invasive subpopulation of RBE cells; iHCCC-9810, invasive subpopulation of HCCC-9810 cells. NG, $\mathrm{P}>0.05 ;{ }^{* * *} \mathrm{P}<0.001 ;{ }^{* * * *} \mathrm{P}<0.0001$; all vs. expressions in non-invasive cell lines, respectively.

and iHCCC-9810 cells with RT-qPCR. As presented in Fig. 1, the levels of miR-101-3p and miR-147a increased significantly $(\mathrm{P}<0.001)$ in the invasive cell subpopulations compared with the normal cell lines, whereas the level of miR-93-3p and miR-26b-5p was significantly decreased $(\mathrm{P}<0.001)$. There was no alteration in the level of miR-196-5p. Therefore, the levels of miR-101-3p, miR-147a, miR-93-3p and miR-26b-5p were selected for quantification in human ICC tissue.

miR-26-5p is downregulated in ICC tumors. To explore the roles of miRNAs in human ICC development, the expression levels of the four previously named candidate miRNAs were detected in 20 samples of ICC tumor and matched adjacent normal tissues with RT-qPCR. Only the expression of miR-26b-5p was significantly downregulated in the tumor tissues compared with the adjacent normal tissues $(\mathrm{P}=0.009$; Fig. 2A), whereas no change was observed in the expression of miR-101-3p, miR-147a and miR-93-3p (Fig. 2B-D).

In addition, to determine whether miR-26b-5p expression was associated with ICC metastasis, miR-26b-5p expression level was compared between tumor tissues from 20 patients with mICC and 20 patients without detected metastases. RT-qPCR revealed that the miR-26b-5p expression levels were significantly lower in metastatic tumors compared with non-metastatic tumors ( $\mathrm{P}=0.005$; Fig. $2 \mathrm{E})$. These results suggested that miR-26b-5p was significantly downregulated in ICC, and that the decreased expression of miR-26b-5p was associated with the metastatic behavior of ICC tumors and cells.

miR-26b-5p regulates ICC cell proliferation, migration and invasion. To determine the functional significance of the aberrant expression of miR-26b-5p in ICC, RBE and iRBE cells were transfected with an miR-26b-5p mimic or inhibitor and the viability of the cells was examined with an MTT assay. As presented in Fig. 3A, compared with RBE cells, the viability in all the iRBE groups was increased. Transfection with an miR-26b-5p mimic reduced the rate of proliferation in the RBE and iRBE cells ( $\mathrm{P}=0.0249$ and $\mathrm{P}=0.0042$, respectively), whereas transfection with an miR-26b-5p inhibitor increased the rate of proliferation in RBE and iRBE cells $(\mathrm{P}=0.0054$ and 
A

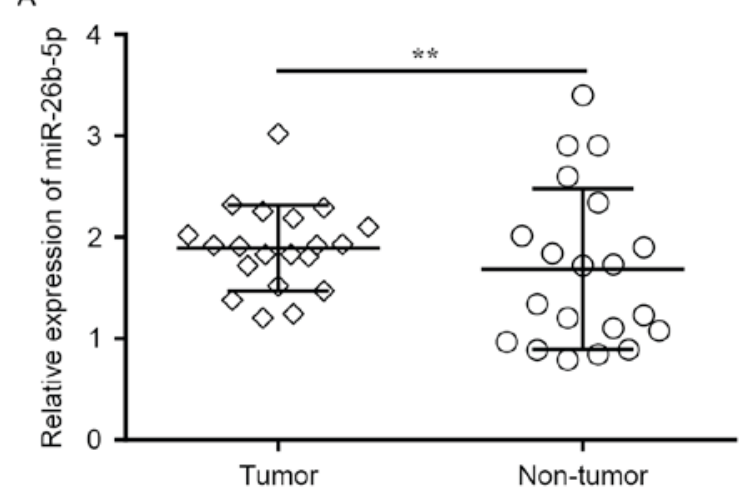

C

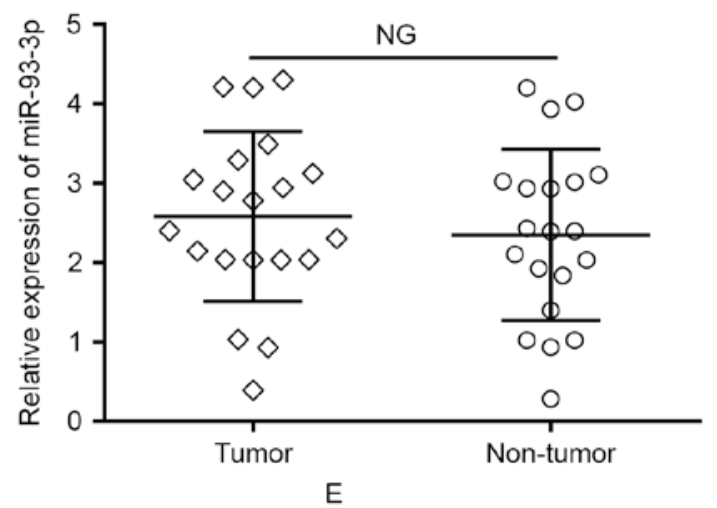

E
B

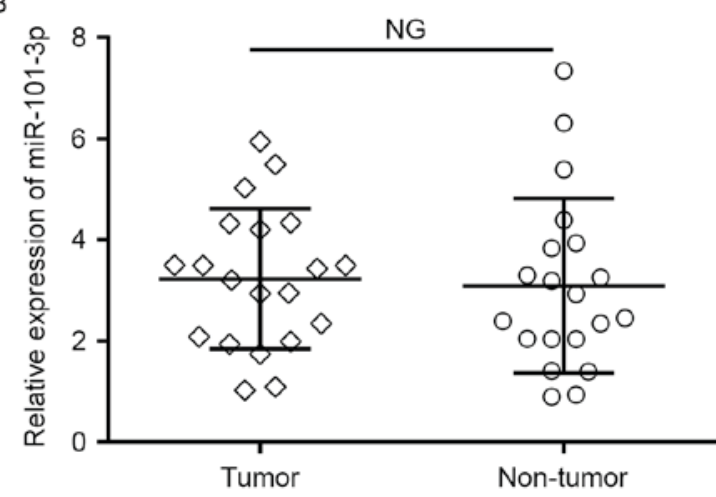

D
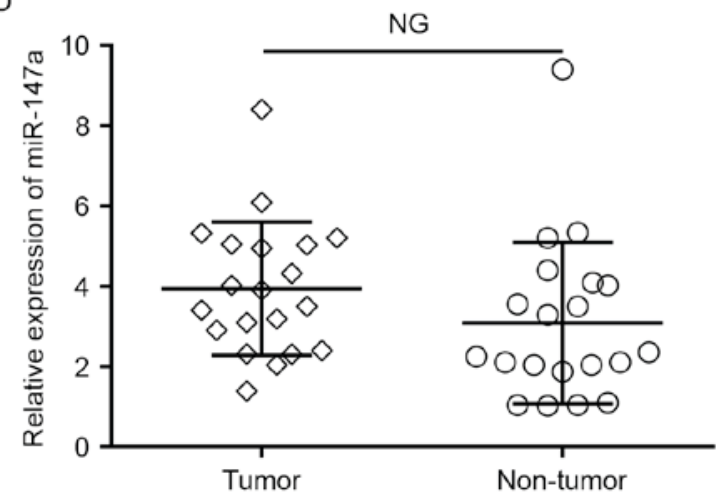

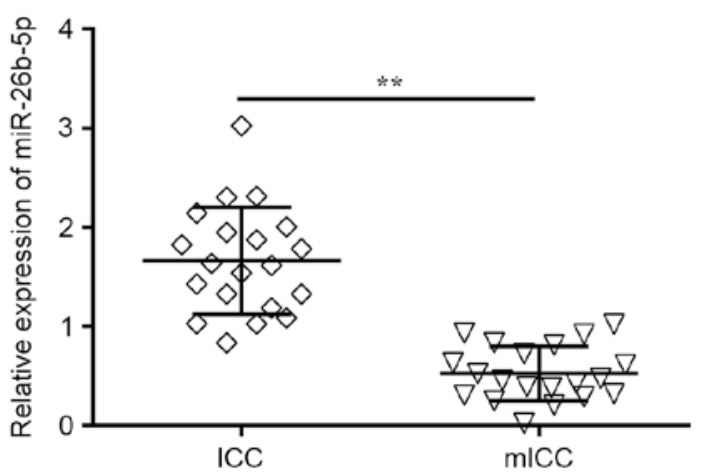

Figure 2. Expression levels of (A) miR-26b-5p, (B) miR-101-3p, (C) miR-93-3p and (D) miR-147a in ICC tumor and paired adjacent normal tissues. (E) Expression levels of miR-26b-5p in ICC and mICC tissues. miR, microRNA; ICC, intrahepatic cholangiocarcinoma; mICC, intrahepatic cholangiocarcinoma with lymphatic metastasis. NG, $\mathrm{P}>0.05 ;{ }^{* *} \mathrm{P}<0.01$; vs. non-tumor issues.

$\mathrm{P}=0.0084$, respectively). This data suggested that miR-26b-5p expression significantly inhibited cell proliferation in the two cell lines.

To confirm this conclusion, pre-miR-26b-5p was transfected into iRBE and iHCCC-9810 cells, and the viability of the cells was examined. The data revealed that transfection with pre-miR-26b-5p significantly inhibited the cell viability compared with transfection with a non-sense control $(\mathrm{P}<0.0001$ and $\mathrm{P}=0.0013$, respectively; Fig. $3 \mathrm{~B})$, which is in accord with the previous data.

In addition to cell proliferation, the effects of miR-26b-5p on ICC cell migration and invasion were also explored. Cells were transfected with an miR-26b-5p mimic or inhibitor, and their migration and invasion abilities were measured with a Transwell assay. As shown in Fig. 3C, compared with the non-sense control group, miR-26b-5p mimics reduced the invasion ability in HCCC-9810 and iHCCC-9810 cells
( $\mathrm{P}=0.0106$ and $\mathrm{P}=0.0051$, respectively); the miR-26b-5p inhibitor, however, increased the cell invasion ability of the cells $(\mathrm{P}=0.0028$ and $\mathrm{P}=0.0162$, respectively). Taken together, miR-26b-5p inhibits the proliferation, migration and invasion of human ICC cells.

miR-26b-5p suppresses ICC migration and invasion by directly targeting S100A7. miRNAs exert their function by regulating the expression of downstream target genes (21-23). In order to identify the underlying mechanisms by which miR-26-5p suppresses ICC invasion, the target prediction software TargetScan was used to search for predicted direct target genes of miR-26b-5p. An initial screening identified S100A7 as a potential direct target of miR-26b-5p, which is a calcium-binding protein previously implicated in cell invasion in a number of types of cancer $(24,25)$. In order to confirm this prediction, S100A7 expression was compared between 

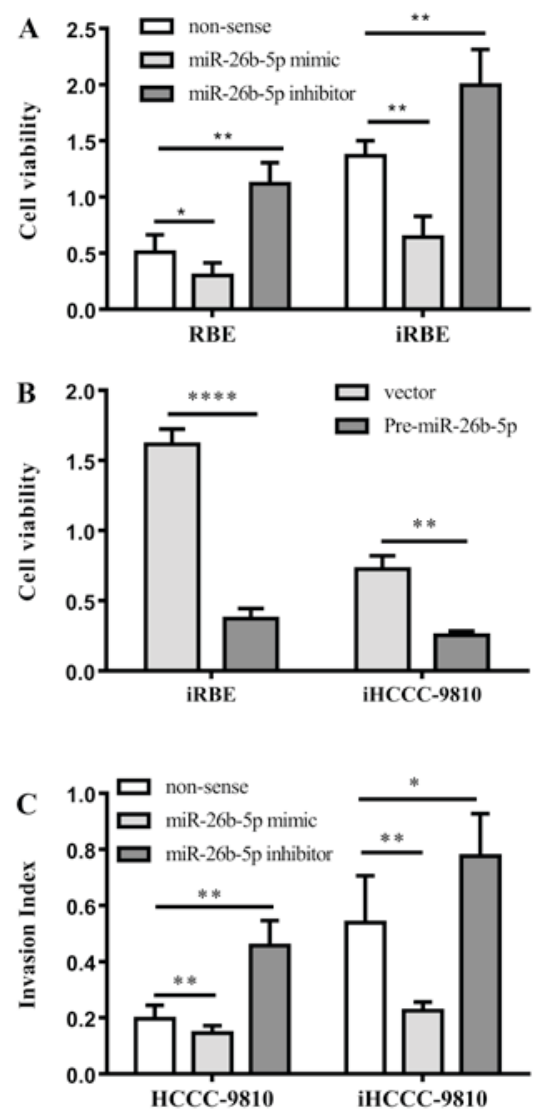

Figure 3. Effects of miR-26b-5p on intrahepatic cholangiocarcinoma cell proliferation, migration and invasion. (A) Viability of RBE and iRBE cells transfected with an miR-26b-5p mimic or inhibitor. (B) Viability of iRBE or iHCCC-9810 cells transfected with a control vector or pre-miR-26b-5p. (C) Extent of invasion by HCCC-9810 or iHCCC-9810 cells following transfection with a control, an miR-26b-5p mimic or an miR-26b-5p inhibitor. miR, microRNA; iRBE, invasive subpopulation of RBE cells; iHCCC-9810, invasive subpopulation of HCCC-9810 cells. ${ }^{*} \mathrm{P}<0.05 ;{ }^{* *} \mathrm{P}<0.01 ;{ }^{* * * *} \mathrm{P}<0.0001$; vs. non-sense vectors respectively.

normal and invasive cell lines. S100A7 was upregulated in iHCCC-9810 and iRBE compared with HCCC-9810 and RBE cells $(\mathrm{P}=0.0002$ and $\mathrm{P}=0.0024$; Fig. 4A). Furthermore, S100A7 expression levels were tested in ICC and mICC tumor samples, and the result indicated that, in line with the result from cell lines, S100A7 expression in mICC tumors was significantly higher compared with that in ICC samples ( $\mathrm{P}=0.0003$; Fig. 4B), indicating that S100A7 expression is inversely associated with miR-26b-5p.

To investigate the interaction between S100A7 and miR-26b-5p, an miR-26b-5p mimic was transfected into iRBE and iHCCC-9810 cells, in which miR-26b-5p expression was low, and the S100A7 expression was measured. The data revealed that, compared with the control group, transfection with an miR-26b-5p mimic significantly lowered the expression of S100A7 ( $\mathrm{P}=0.0002$ and $\mathrm{P}<0.0001$; Fig. 4C). In addition, transfection with pre-miR-26b-5p also inhibited S100A7 expression in the two invasive cell lines $(\mathrm{P}=0.0001$ and $\mathrm{P}<0.0001$; Fig. 4D).

Additionally, an miR-26b-5p inhibitor was transfected into RBE and HCCC-9810 cells, in which the expression of
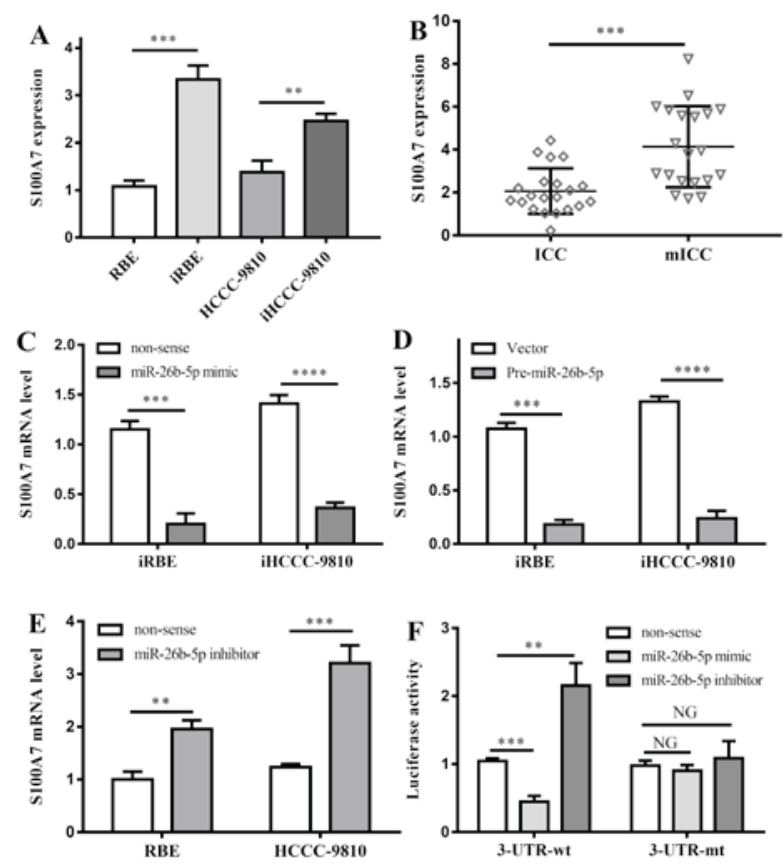

Figure 4. Interactions between S100A7 and miR-26b-5p. (A) S100A7 expression in 4 different kinds of cell lines. ${ }^{* *} \mathrm{P}<0.01,{ }^{* * *} \mathrm{P}<0.001$ vs. non-invasive cell lines, respectively; (B) S100A7 expression in ICC and mICC tumor tissues. ${ }^{* * * *} \mathrm{P}<0.001$ vs. ICC tumor tissues. (C) The effects of miR-26b-5p mimic on invasive cell lines. ${ }^{* * * *} \mathrm{P}<0.001,{ }^{* * * *} \mathrm{P}<0.0001$ vs. non-sense controls. (D) The effects of pre-miR-26b-5p on invasive cell lines. ${ }^{* * *} \mathrm{P}<0.001,{ }^{* * * * *} \mathrm{P}<0.0001$ vs. non-sense controls. (E) The effects of miR-26b-5p inhibitor on RBE and HCCC-9810 cell lines. ${ }^{* *} \mathrm{P}<0.01,{ }^{* * * *} \mathrm{P}<0.001$ vs. non-sense controls. (F) Effects of miR-26b-5p mimic and inhibitor on $3^{\prime} \mathrm{UTR}$ region of S100A7. NG, P>0.05; ${ }^{* *} \mathrm{P}<0.01 ;{ }^{* * *} \mathrm{P}<0.001$; all vs. non-sense control. ICC, intrahepatic cholangiocarcinoma; mICC, ICC with lymphatic metastasis; 3'UTR, 3' untranslated region; S100A7, S100 calcium-binding protein A7; miR, microRNA; iRBE, invasive subpopulation of RBE cells; iHCCC-9810, invasive subpopulation of HCCC-9810 cells.

miR-26b-5p was normal. The data demonstrated that following the transfection with the miR-26b-5p inhibitor, S100A7 in the two cell lines was significantly overexpressed $(\mathrm{P}=0.0019$ and $\mathrm{P}=0.0007$, respectively; Fig. 4E). The results verified that miR-26b-5p was associated with the inhibition of S100A expression.

To determine whether miR-26b-5p binds directly to the 3'UTR region of S100A7 mRNA, a dual-luciferase reporter assay was performed. Transfection with an miR-26b-5p mimic markedly reduced the luciferase activity of the 3'UTR-wt S100A7, whereas an increase in the luciferase activity of the 3'UTR-wt S100A7 was observed following the transfection of an miR-26b-5p inhibitor $(\mathrm{P}=0.0004$ and $\mathrm{P}=0.0038$, respectively; Fig. 4F). However, no significant difference in the luciferase activity of 3'UTR-mt S100A7 was observed following the transfection with the miR-26b-5p mimic or inhibitor (Fig. 4F).

It was also investigated whether the interaction between miR-26b-5p and S100A7 led to alterations in the migration and invasion ability of ICC cells. The non-sense control, the miR-26b-5p mimic, the miR-26b-5p mimic with the 3'UTR-wt S100A7 vector or the miR-26b-5p mimic with the 3'UTR-wt S100A7 vector were transfected into iRBE and iHCCC-9810 cells, and their invasive ability was assessed with a Transwell 

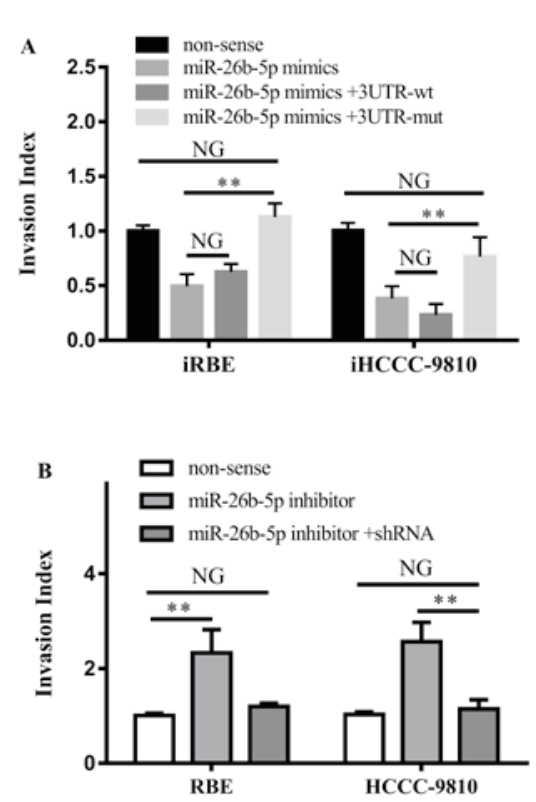

Figure 5. Effects of the interaction between S100A7 and miR-26b-5p on cel invasion. (A) Invasiveness of iRBE or iHCCC-9810 cells following transfection with a non-sense control, or an miR-25b-5p mimic with a 3'UTR wt or mt S100A7. (B) Invasiveness of RBE and HCCC-9810 cells following transfection with a non-sense control, an miR-26b-5p inhibitor or the inhibitor and shRNA against S100A7. S100A7, S100 calcium-binding protein A7; miR, microRNA; iRBE, invasive subpopulation of RBE cells; iHCCC-9810, invasive subpopulation of HCCC-9810 cells; 3'UTR, 3' untranslated region; wt, wild-type; mt, mutant; shRNA, short hairpin RNA. NG, $\mathrm{P}>0.05 ;{ }^{* * *} \mathrm{P}<0.01$; vs non-sense controls respectively.

invasion assay. The results demonstrated that the invasion ability of cells transfected with the miR-26b-5p mimic and 3'UTR-wt vector was not altered compared with cells transfected with the miR-26b-5p mimic alone, whereas transfection with the miR-26b-5p mimic and the 3'UTR-mt vector increased the invasion ability (iRBE, $\mathrm{P}=0.0026$; iHCCC-9810, $\mathrm{P}=0.0054)$ and returned it to similar levels as the non-sense control cells (Fig. 5A).

Furthermore, the miR-26b-5p inhibitor or the miR-26b-5p inhibitor with a small hairpin RNA against S100A7 were transfected into RBE and HCCC-9810 cells. It was revealed that transfection with the miR-26b-5p inhibitor increased the invasion ability of the two cells $(\mathrm{P}=0.0097$ and $\mathrm{P}=0.0027$, respectively), whereas transfection with the inhibitor and the shRNA against S100A7 did not change the invasiveness of the cells (Fig. 5B). Therefore, the results demonstrated that miR-26b-5p suppressed the invasive ability of ICC cells by directly targeting the 3'UTR region of S100A7 mRNA.

\section{Discussion}

A number of studies have demonstrated that miRNAs regulate the expression of oncogenes and tumor suppressors, which may be associated with the initiation and development of HCC and ICC $(11,14,17)$. The expression levels of five miRNAs potentially associated with cancer development were investigated in ICC cells, and four candidate miRNAs were selected on the basis of their differential expression in invasive cell subpopulations. These candidate miRNAs were further examined in 20 paired ICC and matched tumor-adjacent tissues with RT-qPCR. The data revealed that only the expression of miR-26b-5p in ICC was significantly lower than in matched tumor-adjacent tissues. The expression of miR-26b-5p was also significantly reduced in mICC tissue compared with non-metastatic ICC tumors. The abnormal express of miR-26b-5p has been demonstrated in types of cancer, including pulmonary and cancer $(26,27)$. However, to the best of our knowledge, the present study was the first to identify that miR-26b-5p exhibits a function in HCC.

Gain- and loss-of-function experiments demonstrated that an miR-26b-5p mimic inhibited the proliferation, migration and invasion of RBE and HCCC-9810 cells, whereas an miR-26b-5p inhibitor led an increase in RBE and HCCC-9810 cell proliferation, migration and invasion. These results indicated that miR-26b-5p inhibited the proliferation, migration and invasion of ICC cells. TargetScan was utilized to predict the target genes of miR-26b-5p, and it was predicted that S100A7 may be a direct target. In order to confirm this prediction, the expression level of S100A7 was determined in RBE, iRBE, HCCC-9810 and iHCCC-9810 cells, and in non-metastatic ICC and mICC tumors. The data revealed that S100A7 was upregulated in the two invasive cells and mICC tumors compared with that in cell lines and non-metastatic ICC tumors. Subsequently, the effect of the miR-26b-5p mimic or inhibitor on S100A7 expression was determined, and S100A7 expression was demonstrated to be inversely associated with miR-26b-5p expression in ICC. Furthermore, the effect of the interaction between miR-26b-5p and S100A7 on the invasive ability of the cells was investigated. These results demonstrated that the miR-26b-5p mimic decreased the invasive ability of the invasive cell sub-populations by downregulating S100A7 expression, whereas the miR-26b-5p inhibitor increased the invasion ability of the RBE and HCCC-9810 cells by upregulating S100A7 expression. Therefore, to the best of our knowledge, it was demonstrated for the first time that miR-26b-5p inhibits cell migration and invasion by suppressing S100A7 expression in ICC.

In summary, the present study revealed that miR-26b-5p was downregulated in ICC tissues and cells, particularly in invasive ICC subpopulations and tumors from patients with mICC. In addition, it was revealed that miR-26b-5p inhibited the proliferation, migration and invasion of ICC cells by targeting S100A7. In conclusion, the present study demonstrated that miR-26b-5p functions as a tumor suppressive miRNA, and inhibits the invasive behavior of ICC by inhibiting S100A7.

\section{Acknowledgements}

The present study was supported by a grant from the Natural Science Foundation of PRC (grant no. 81072026).

\section{References}

1. Bartella I and Dufour JF: Clinical diagnosis and staging of intrahepatic cholangiocarcinoma. J Gastrointestin Liver Dis 24: 481-489, 2015.

2. Guo LH and Xu HX: Contrast-enhanced ultrasound in the diagnosis of hepatocellular carcinoma and intrahepatic cholangiocarcinoma: Controversy over the ASSLD guideline. Biomed Res Int 2015: 349172, 2015 . 
3. Lafaro KJ, Cosgrove D, Geschwind JF, Kamel I, Herman JM and Pawlik TM: Multidisciplinary care of patients with intrahepatic cholangiocarcinoma: Updates in management. Gastroenterol Res Pract 2015: 860861, 2015.

4. Brandi G, Venturi M, Pantaleo MA and Ercolani G; GICO Cholangiocarcinoma: Current opinion on clinical practice diagnostic and therapeutic algorithms: A review of the literature and a long-standing experience of a referral center. Dig Liver Dis 48 : 231-241, 2016

5. Maithel SK, Gamblin TC, Kamel I, Corona-Villalobos CP, Thomas M and Pawlik TM: Multidisciplinary approaches to intrahepatic cholangiocarcinoma. Cancer 119: 3929-3942, 2013.

6. Hyder O, Hatzaras I, Sotiropoulos GC, Paul A, Alexandrescu S, Marques H, Pulitano C, Barroso E, Clary BM, Aldrighetti L, et al: Recurrence after operative management of intrahepatic cholangiocarcinoma. Surgery 153: 811-818, 2013.

7. Ambros V: The functions of animal microRNAs. Nature 431: 350-355, 2004

8. Bartel DP: MicroRNAs: Genomics, biogenesis, mechanism, and function. Cell 116: 281-297, 2004.

9. Hawkins PG and Morris KV: RNA and transcriptional modulation of gene expression. Cell Cycle 7: 602-607, 2008.

10. Kunej T, Godnic I, Ferdin J, Horvat S, Dove P and Calin GA Epigenetic regulation of microRNAs in cancer: An integrated review of literature. Mutat Res 717: 77-84, 2011.

11. Haga H, Yan I, Takahashi K, Wood J and Patel T: Emerging insights into the role of microRNAs in the pathogenesis of cholangiocarcinoma. Gene Expr 16: 93-99, 2014.

12. Maemura K, Natsugoe S and Takao S: Molecular mechanism of cholangiocarcinoma carcinogenesis. J Hepatobiliary Pancreat Sci 21: 754-760, 2014

13. Piontek K and Selaru FM: MicroRNAs in the biology and diagnosis of cholangiocarcinoma. Semin Liver Dis 35: 55-62, 2015.

14. Chinese Medical Association: The updated grading standards for primary hepatic cancer. In: Clinical Practice Guidelines - Tumor. People's Medical Publishing House, China, pp325-326, 2001.

15. Livak KJ and Schmittgen TD: Analysis of relative gene expression data using real-time quantitative PCR and the 2(-Delta Delta C(T)) method. Methods 25: 402-408, 2001.

16. Sheng Y, Li J, Zou C, Wang S, Cao Y, Zhang J, Huang A and Tang H: Downregulation of miR-101-3p by hepatitis B virus promotes proliferation and migration of hepatocellular carcinoma cells by targeting Rab5a. Arch Virol 159: 2397-2410, 2014
17. Augello C, Gianelli U, Savi F, Moro A, Bonoldi E, Gambacorta M, Vaira V, Baldini L and Bosari S: MicroRNA as potential biomarker in $\mathrm{HCV}$-associated diffuse large B-cell lymphoma. J Clin Pathol 67: 697-701, 2014.

18. Ertekin S, Yildirim O, Dinc E, Ayaz L, Fidanci SB and Tamer L: Evaluation of circulating miRNAs in wet age-related macular degeneration. Mol Vis 20: 1057-1066, 2014.

19. Kozubek J, Ma Z, Fleming E, Duggan T, Wu R, Shin DG and Dadras SS: In-depth characterization of microRNA transcriptome in melanoma. PLoS One 8: e72699, 2013.

20. Yan S, Wang J, Zhang W and Dai J: Circulating MicroRNA profiles altered in mice after 28 days exposure to perfluorooctanoic acid. Toxicol Lett: Oct 24, 2013 (Epub ahead of print).

21. Tessitore A, Cicciarelli G, Del Vecchio F, Gaggiano A, Verzella D, Fischietti M, Vecchiotti D, Capece D, Zazzeroni F and Alesse E: MicroRNAs in the DNA damage/repair network and cancer. Int J Genomics 2014: 820248, 2014.

22. Son DJ, Kumar S, Takabe W, Kim CW, Ni CW, Alberts-Grill N, Jang IH, Kim S, Kim W, Won Kang S, et al: The atypical mechanosensitive microRNA-712 derived from pre-ribosomal RNA induces endothelial inflammation and atherosclerosis. Nat Commun 4: 3000, 2013.

23. Sampath D, Liu C, Vasan K, Sulda M, Puduvalli VK, Wierda WG and Keating MJ: Histone deacetylases mediate the silencing of miR-15a, miR-16, and miR-29b in chronic lymphocytic leukemia. Blood 119: 1162-1172, 2012.

24. Dey KK, Sarkar S, Pal I, Das S, Dey G, Bharti R, Banik P, Ray JG, Maity S, Kulavi I and Mandal M: Erratum to: Mechanistic attributes of S100A7 (psoriasin) in resistance of anoikis resulting tumor progression in squamous cell carcinoma of the oral cavity. Cancer Cell Int 15: 94, 2015.

25. Liu G, Wu Q, Liu G, Song X and Zhang J: Psoriasin (S100A7) is a novel biomarker for lung squamous cell carcinoma in humans. Cancer Cell Int 15: 18, 2015.

26. Wu T, Chen W, Liu S, Lu H, Wang H, Kong D, Huang X, Kong Q, Ning Y and Lu Z: Huaier suppresses proliferation and induces apoptosis in human pulmonary cancer cells via upregulation of miR-26b-5p. FEBS Lett 588: 2107-2114, 2014.

27. Armstrong DA, Green BB, Seigne JD, Schned AR and Marsit CJ: MicroRNA molecular profiling from matched tumor and bio-fluids in bladder cancer. Molecular Cancer 14: 194. 2015. 